\title{
Remark on Lower Bound for Forgotten Topological Index
}

\author{
E. I. Milovanović, M. M. Matejić, I. Ž. Milovanović
}

\begin{abstract}
Let $G$ be a simple connected graph with $n$ vertices and $m$ edges with vertex degree sequence $d_{1} \geq d_{2} \geq \cdots \geq d_{n}>0$. Denote by $F=\sum_{i=1}^{n} d_{i}^{3}$ forgotten topological index of graph $G$. In this paper we give some lower bounds for invariant $F$. Also, obtained bounds are compared with some known bounds from the literature.
\end{abstract}

Keywords: Vertex degree, the first Zagreb index, forgotten topological index

\section{Introduction}

Let $G$ be a simple connected graph with $n$ vertices and $m$ edges. Denote by $d_{1} \geq d_{2} \geq \cdots \geq$ $d_{n}>0$ a sequence of vertex degrees of graph $G$. Throughout this paper we use standard notation: $\Delta=d_{1}, \Delta_{2}=d_{2}$, and $\delta=d_{n}$.

In [5] vertex-degree-based topological indices, named the first and the second Zagreb indices $M_{1}$ and $M_{2}$, were defined as

$$
M_{1}=M_{1}(G)=\sum_{i=1}^{n} d_{i}^{2} \quad \text { and } \quad M_{2}=M_{2}(G)=\sum_{i \sim j} d_{i} d_{j}
$$

where $i \sim j$ denotes the adjacency of the vertices $i$ and $j$ in graph $G$.

Details on these topological indices can be found in $[1,2,6,7]$.

In [4] (see also [6]) forgotten topological index $F$ was defined as

$$
F=F(G)=\sum_{i=1}^{n} d_{i}^{3} .
$$

Let $E=\left\{e_{1}, e_{2}, \ldots, e_{m}\right\}$ be a set of edges of graph $G$ and $d\left(e_{1}\right) \geq d\left(e_{2}\right) \geq \cdots \geq d\left(e_{m}\right)$ sequence of edge degrees. In [10], an edge-degree graph topological index, named refor-

Manuscript received September 12, 2016; accepted January 23, 2017.

E. I. Milovanović, M. M. Matejić, I. Ž. Milovanović are with the Faculty of Electronic Engineering, Niš, Serbia 
mulated Zagreb index, $E M_{1}$, is defined as

$$
E M_{1}=E M_{1}(G)=\sum_{i=1}^{m} d\left(e_{i}\right)^{2} .
$$

Of course, it is easy to note that $E M_{1}$ is not new topological index, since it is the first Zagreb index for a line-graph $L=L(G)$ of graph $G$.

In this paper we state two inequalities that set lower bounds for invariant $F$ in terms of topological index $M_{1}$ and graph parameters $m, \Delta, \Delta_{2}$, and $\delta$. Obtained results will be used to determine lower bounds for topological indices $E M_{1}$ and $M_{2}$.

\section{Preliminaries}

In this section we give some known results for invariants $F, M_{1}$ and $E M_{1}$ that will be needed in the subsequent considerations.

In [4] the following inequality for graph invariant $F$ was proved

$$
F \geq \frac{M_{1}^{2}}{2 m}
$$

with equality if and only if $G$ is a regular graph.

The following equality was proved in [15] for graph invariant $E M_{1}$

$$
E M_{1}=F+2 M_{2}-4 M_{1}+4 m \text {. }
$$

In [11] it was proved

$$
E M_{1} \geq \frac{M_{1}^{2}}{2 m}+2 M_{2}-4 M_{1}+4 m .
$$

Equality holds if and only if $L(G)$ is regular.

In [9] it was proved

$$
F \leq \frac{\Delta^{2}+\delta^{2}}{\Delta \delta} M_{2}
$$

\section{Main result}

The following theorem establishes lower bound for invariant $F$ in terms of topological index $M_{1}$ and graph parameters $m, \Delta$ and $\Delta_{2}$.

Theorem 3.1. Let $G$ be a simple connected graph with $n, n \geq 2$, vertices and $m$ edges. Then

$$
F \geq \frac{M_{1}^{2}}{2 m}+\frac{\Delta \Delta_{2}\left(\Delta-\Delta_{2}\right)^{2}}{2 m} .
$$

Equality holds if and only if $G$ is regular graph. 
Proof. Let $p=\left(p_{i}\right), i=1,2, \ldots, m$, be positive real number sequence, and $a=\left(a_{i}\right)$ and $b=\left(b_{i}\right), i=1,2, \ldots, m$, sequences of non-negative real numbers of similar monotonicity. In [14] (see also [13]) it was proved that

$$
T_{n}(a, b ; p) \geq T_{n-1}(a, b ; p), \quad n \geq 2,
$$

where

$$
T_{n}(a, b ; p)=\sum_{i=1}^{n} p_{i} \sum_{i=1}^{n} p_{i} a_{i} b_{i}-\sum_{i=1}^{n} p_{i} a_{i} \sum_{i=1}^{n} p_{i} b_{i} .
$$

From (6) it follows

$$
T_{n}(a, b ; p) \geq T_{n-1}(a, b ; p) \geq \cdots \geq T_{2}(a, b ; p) \geq 0 .
$$

Since

$$
\begin{aligned}
T_{2}(a, b ; p) & =\sum_{i=1}^{2} p_{i} \sum_{i=1}^{2} p_{i} a_{i} b_{i}-\sum_{i=1}^{2} p_{i} a_{i} \sum_{i=1}^{2} p_{i} b_{i} \\
& =\left(p_{1}+p_{2}\right)\left(p_{1} a_{1} b_{1}+p_{2} a_{2} b_{2}\right)-\left(p_{1} a_{1}+p_{2} a_{2}\right)\left(p_{1} b_{1}+p_{2} b_{2}\right) \\
& =p_{1} p_{2}\left(a_{1}-a_{2}\right)\left(b_{1}-b_{2}\right)
\end{aligned}
$$

we have that

$$
\sum_{i=1}^{n} p_{i} \sum_{i=1}^{n} p_{i} a_{i} b_{i} \geq \sum_{i=1}^{n} p_{i} a_{i} \sum_{i=1}^{n} p_{i} b_{i}+p_{1} p_{2}\left(a_{1}-a_{2}\right)\left(b_{1}-b_{2}\right) .
$$

For $p_{i}=a_{i}=b_{i}=d_{i}, i=1,2, \ldots, n$, this inequality becomes

$$
\sum_{i=1}^{n} d_{i} \sum_{i=1}^{n} d_{i}^{3} \geq\left(\sum_{i=1}^{n} d_{i}^{2}\right)^{2}+d_{1} d_{2}\left(d_{1}-d_{2}\right)^{2}
$$

wherefrom we get (5).

Remark 3.2. Since

the inequality (5) is stronger than (1).

$$
\frac{M_{1}^{2}}{2 m}+\frac{\Delta \Delta_{2}\left(\Delta-\Delta_{2}\right)^{2}}{2 m} \geq \frac{M_{1}^{2}}{2 m}
$$

Corollary 3.3. Let $G$ be a simple connected graph with $n, n \geq 2$, vertices and $m$ edges. Then

$$
F \geq \frac{8 m^{3}}{n^{2}}+\frac{\Delta \Delta_{2}\left(\Delta-\Delta_{2}\right)^{2}}{2 m},
$$

with equality if and only if $G$ is regular graph. 
Proof. Inequality (8) is a direct consequence of (5) and the following inequality

$$
M_{1} \geq \frac{4 m^{2}}{n},
$$

proved in [3].

Corollary 3.4. Let $G$ be a simple connected graph with $n, n \geq 2$, vertices and $m$ edges. Then

$$
M_{2} \geq \frac{\Delta \delta}{2 m\left(\Delta^{2}+\delta^{2}\right)}\left(M_{1}^{2}+\Delta \Delta_{2}\left(\Delta-\Delta_{2}\right)^{2}\right),
$$

with equality if and only if $G$ is regular graph.

Corollary 3.5. Let $G$ be a simple connected graph with $n, n \geq 2$, vertices and $m$ edges. Then

$$
E M_{1} \geq \frac{M_{1}^{2}}{2 m}+2 M_{2}-4 M_{1}+4 m+\frac{\Delta \Delta_{2}\left(\Delta-\Delta_{2}\right)^{2}}{2 m}
$$

with equality if and only if $G$ is regular.

Remark 3.6. Since

$$
\frac{\Delta \Delta_{2}\left(\Delta-\Delta_{2}\right)^{2}}{2 m} \geq 0
$$

the inequality (11) is stronger than (3).

Remark 3.7. Note that inequality (7) is a generalization of Chebyshev inequality (see for example [12]).

Theorem 3.8. Let $G$ be a simple connected graph with $n, n \geq 3$, vertices and $m$ edges. Then

$$
F \geq \delta^{3}+\frac{\left(M_{1}-\delta^{2}\right)^{2}}{2 m-\delta}+\frac{\Delta \Delta_{2}\left(\Delta-\Delta_{2}\right)^{2}}{2 m-\delta} .
$$

Equality holds if and only if $G$ is regular graph.

Proof. According to (7) we have that

$$
\sum_{i=1}^{n-1} p_{i} \sum_{i=1}^{n-1} p_{i} a_{i} b_{i} \geq \sum_{i=1}^{n-1} p_{i} a_{i} \sum_{i=1}^{n-1} p_{i} b_{i}+p_{1} p_{2}\left(a_{1}-a_{2}\right)\left(b_{1}-b_{2}\right) .
$$

Putting $p_{i}=a_{i}=b_{i}=d_{i}, i=1,2, \ldots, n-1$, in this inequality, we get

$$
\sum_{i=1}^{n-1} d_{i} \sum_{i=1}^{n-1} d_{i}^{3} \geq\left(\sum_{i=1}^{n-1} d_{i}^{2}\right)^{2}+d_{1} d_{2}\left(d_{1}-d_{2}\right)^{2},
$$

i.e.

$$
(2 m-\delta)\left(F-\delta^{3}\right) \geq\left(M_{1}-\delta^{2}\right)^{2}+\Delta \Delta_{2}\left(\Delta-\Delta_{2}\right)^{2},
$$

wherefrom we obtain inequality (12). 
Corollary 3.9. Let $G$ be a simple connected graph with $n, n \geq 3$, vertices and $m$ edges. Then

$$
F \geq 2 m \delta^{2}+\frac{\Delta \Delta_{2}\left(\Delta-\Delta_{2}\right)^{2}}{2 m-\delta}
$$

with equality if and only if $G$ is regular.

Corollary 3.10. Let $G$ be a simple connected graph with $n, n \geq 3$, vertices and $m$ edges. Then

$$
M_{2} \geq \frac{\Delta \delta}{\Delta^{2}+\delta^{2}}\left(2 m \delta^{2}+\frac{\Delta \Delta_{2}\left(\Delta-\Delta_{2}\right)^{2}}{2 m-\delta}\right) .
$$

Corollary 3.11. Let $G$ be a simple connected graph with $n, n \geq 3$, vertices and $m$ edges. Then

$$
E M_{1} \geq \delta^{3}+\frac{\left(M_{1}-\delta^{2}\right)^{2}}{2 m-\delta}+\frac{\Delta \Delta_{2}\left(\Delta-\Delta_{2}\right)^{2}}{2 m-\delta}+2 M_{2}-4 M_{1}+4 m,
$$

with equality if and only if $G$ is regular.

Theorem 3.12. Let $G$ be a simple connected graph with $n, n \geq 2$, vertices and $m$ edges. Then

$$
M_{1} \geq \frac{4 m^{2}}{n}+\frac{\left(\Delta-\Delta_{2}\right)^{2}}{n} .
$$

Equality holds if and only if $G$ is regular graph.

Proof. For $p_{i}=1, a_{i}=b_{i}=d_{i}, i=1,2, \ldots, n$, inequality (7) becomes

$$
n \sum_{i=1}^{n} d_{i}^{2} \geq\left(\sum_{i=1}^{n} d_{i}\right)^{2}+\left(\Delta-\Delta_{2}\right)^{2}
$$

i.e.

$$
n M_{1} \geq 4 m^{2}+\left(\Delta-\Delta_{2}\right)^{2}
$$

wherefrom we obtain (13).

Remark 3.13. Since $\left(\Delta-\Delta_{2}\right)^{2} \geq 0$, the inequality (13) is stronger than (9).

By a similar procedure as in case of Theorem 3.12, the following statement can be proved.

Theorem 3.14. Let $G$ be a simple connected graph with $n, n \geq 3$, vertices and $m$ edges. Then

$$
M_{1} \geq \delta^{2}+\frac{(2 m-\delta)^{2}+\left(\Delta-\Delta_{2}\right)^{2}}{n-1} .
$$

Equality holds if and only if $G$ is regular. 


\section{References}

[1] B. Borovićanin, K. C. Das, B. Furtula, I. Gutman, Zagreb indices: Bounds and Extremal graphs, In: Bounds in Chemical Graph Theory - Basics, (I. Gutman, B. Furtula, K. C. Das, E. Milovanović, I. Milovanović, Eds.), Mathematical Chemistry Monographs, MCM 19, Univ. Kragujevac, Kragujevac, 2017, pp. 67-153.

[2] B. Borovićanin, K. C. DAs, B. Furtula, I. Gutman, Bounds for Zagreb indices, MATCH Commun. Math. Comput. Chem., 78 (2017) 17-100.

[3] C. S. EDWARDS, The largest vertex degree sum for a triangle in a graph, Bull. London Math. Soc., 9 (1977) 203-208.

[4] B. Furtula, I. Gutman, A forgotten topological index, J. Math. Chem., 53 (2015) 11841190.

[5] I. Gutman, N. TRinajstić, Graph theory and molecular orbitals. Total $\pi$-electron energy of alternant hydrocarbons, Chem. Phys. Lett., 17 (1972), 535-538.

[6] I. Gutman, On the origin of two degree-based topological indices, Bull. Acad. Serbie Sci. Arts (Ch. Sci. Math. Natur.), 146 (2014) 39-52.

[7] I. Gutman, K. C. DAS, The first Zagreb index 30 years after, MATCH Commun. Math. Comput. Chem., 50 (2004) 83-92.

[8] I. Gutman, B. Furtula, Ž. Kovijanić Vukićević, G. Popivoda, On Zagreb indices and coindices, MATCH Commun. Math. Comput. Chem., 74 (2015) 5-16.

[9] B. LiU, I. Gutman, On general Randić indices, MATCH Commun. Math. Comput. Chem., 58 (2007) 147-154.

[10] A. Milićević, S. Nikolić, N. Trinajstić, On reformulated Zagreb indices, Mol. Divers., 8 (2004) 393-399.

[11] E. I. Milovanović, I. Ž. Milovanović, E. Ć. Dolićanin, E. Glogić, A note on the first reformulated Zagreb index, Appl. Math. Comput., 273 (2016) 16-20.

[12] D. S. Mitrinović, P. M. VAsić, Analytic inequalities, Springer Verlag, Berlin-HeidelbergNew York, 1970.

[13] D. S. Mitrinović, P. M. VAsić, History, variations and generalisations of the Čebyšev inequality and the question of some priorities, Univ. Beograd Publ. Elektrotehn. Fak. Ser. Mat. Fiz., 461-497 (1974), 1-30.

[14] P. M. VAsić, R. Ž. DJordjević, Čebyšev inequality for convex sets, Univ. Beograd Publ. Elektrotehn. Fak. Ser. Mat. Fiz., 412-460 (1973), 17-20.

[15] B. Zhou, N. Trinajstić, Some properties of the reformulated Zagreb indices, J. Math. Chem., 48 (2010), 714-719. 